\title{
ESTUDO E ANALÍSE DE ESCOAMENTO MONOFÁSICO E BIFÁSICO GÁS-LÍQUIDO EM DUTO ANULAR ATRAVÉS DE PIV
}

\author{
V. E. C. BAPTISTELlA ${ }^{1}$, M. M. H. CELY ${ }^{1}$, A. B. F. DE OLIVEIRA ${ }^{1}$ e O. M. H. \\ RODRÍGUEZ ${ }^{1}$
}

${ }^{1}$ Laboratório de Escoamentos Multifásicos Industriais (LEMI), Departamento de Engenharia Mecânica, Escola de Engenharia de São Carlos, Universidade de São Paulo (USP) victor.baptistella@usp.br

\begin{abstract}
RESUMO - Um estudo teórico-experimental é apresentado, onde escoamentos monofásicos de água, e bifásicos de ar-água são conduzidos num duto anular de $10,5 \mathrm{~m}$ de comprimento, com diâmetro externo de $155 \mathrm{~mm}$ e interno de $60 \mathrm{~mm}$. O duto foi inclinado em $2^{\circ}$ em relação à horizontal para os escoamentos monofásicos, e em $5^{\circ}$ para os bifásicos. A técnica PIV (Particle Image Velocimetry) foi empregada para a obtenção de perfis de velocidade da fase aquosa, bem como estatísticas de turbulência, como: flutuações de velocidade e tensões de Reynolds. Uma comparação com o modelo analítico para o escoamento laminar $(\operatorname{Re}=742)$ foi realizada, a fim de validar as medições da bancada experimental; para os turbulentos $(\operatorname{Re}=7106$ a $R e=20431)$ não há modelos analíticos. Resultados preliminares para os escoamentos bifásicos são expostos $\left(j_{\mathrm{w}}\right.$ $=0,153 \mathrm{~m} / \mathrm{s}$ e $\mathrm{j}_{\mathrm{a}}=0,039 \mathrm{~m} / \mathrm{s}$ ) e os efeitos que a passagem da bolha de ar ocasiona no perfil de velocidades da água são mostrados.
\end{abstract}

Palavras-chaves: PIV, Duto Anular, Escoamento Bifásico, Perfis de Velocidade.

\section{INTRODUÇÃO}

O presente trabalho descreve a aplicação de Velocimetria por Imagem de Partículas (PIV) em escoamentos monofásicos laminares e turbulentos, e em escoamentos bifásicos, arágua, em um duto de geometria anular de grandes dimensões. Perfis de velocidade são obtidos, bem como suas flutuações e Tensões de Reynolds. PIV é uma técnica não intrusiva para a obtenção instantânea de um grande número de vetores de velocidade dentro de um plano de um escoamento. A técnica permite que a estrutura espacial do campo de velocidade seja visualizada, bem como quantificada (Willert, 1999). Em um fluido opticamente homogêneo não há interação significativa da luz incidente com o fluido, tal como refração, assim, informações do campo de velocidade do escoamento podem ser obtidas. No PIV, o movimento do fluido é feito visível pela adição de pequenas partículas, os traçadores, e a partir de suas posições em dois instantes de tempo o deslocamento é obtido. Com estes dados, é possível inferir o campo de velocidades (Westerweel, 1997).

Escoamentos bifásicos em dutos anulares são presentes em aplicações nas indústrias de refrigeração, nuclear e petróleo, porém muitas de suas características não são entendidas 
completamente, sendo importante a aplicação da técnica do PIV em seus estudos. Trabalhos em escoamentos água-óleo, em um tubo horizontal, foram realizados por (Elseth, 2001), (Kumara et al., 2010a), (Kumara et al., 2010b) onde foram analisadas estatísticas como: a distribuição de velocidade axial, parâmetros de flutuações na velocidade, intensidade de turbulência e tensões de Reynolds. (Birvalski et al., 2016), realizaram um estudo sobre escoamento estratificado-ondulado, ar-água, também através de uma tubulação horizontal. Trabalhos similares utilizando PIV em escoamentos bifásicos em dutos anulares não foram encontrados na literatura.

\section{METODOLOGIA}

\subsection{Aparato Experimental Duto Anular}

Os experimentos foram realizados no Laboratório de Escoamentos Multifásicos Industriais (LEMI) da Escola de Engenharia de São Carlos, da Universidade de São Paulo (USP). Para a instalação do sistema de PIV na seção de teste foi utilizado um tanque de retorno (water PIV) como um tanque de água, ver Fig. 1. Este ciclo de escoamento secundário foi responsável por retornar as partículas traçadoras (esferas de vidro oco de $10 \mu \mathrm{m}$ de diâmetro) e permitiu a aquisição das imagens com maior precisão. A seção de teste com 10,5 $\mathrm{m}$ de comprimento é instalada sobre uma viga treliçada. Ela é formada por dois tubos montados concentricamente, os externos de vidro de borosilicato e os internos de PVC. O tubo externo tem um diâmetro interno de $155 \mathrm{~mm}$ e o interno, um diâmetro externo de 60 $\mathrm{mm}$. Uma linha de by-pass permite o uso da válvula de fechamento rápido (QCV) para medir a fração de vazio in situ. A água é mantida em tanques de polietileno. $\mathrm{O}$ ar é armazenado em um tanque pressurizado.

Figura 1 - Aparato Experimental

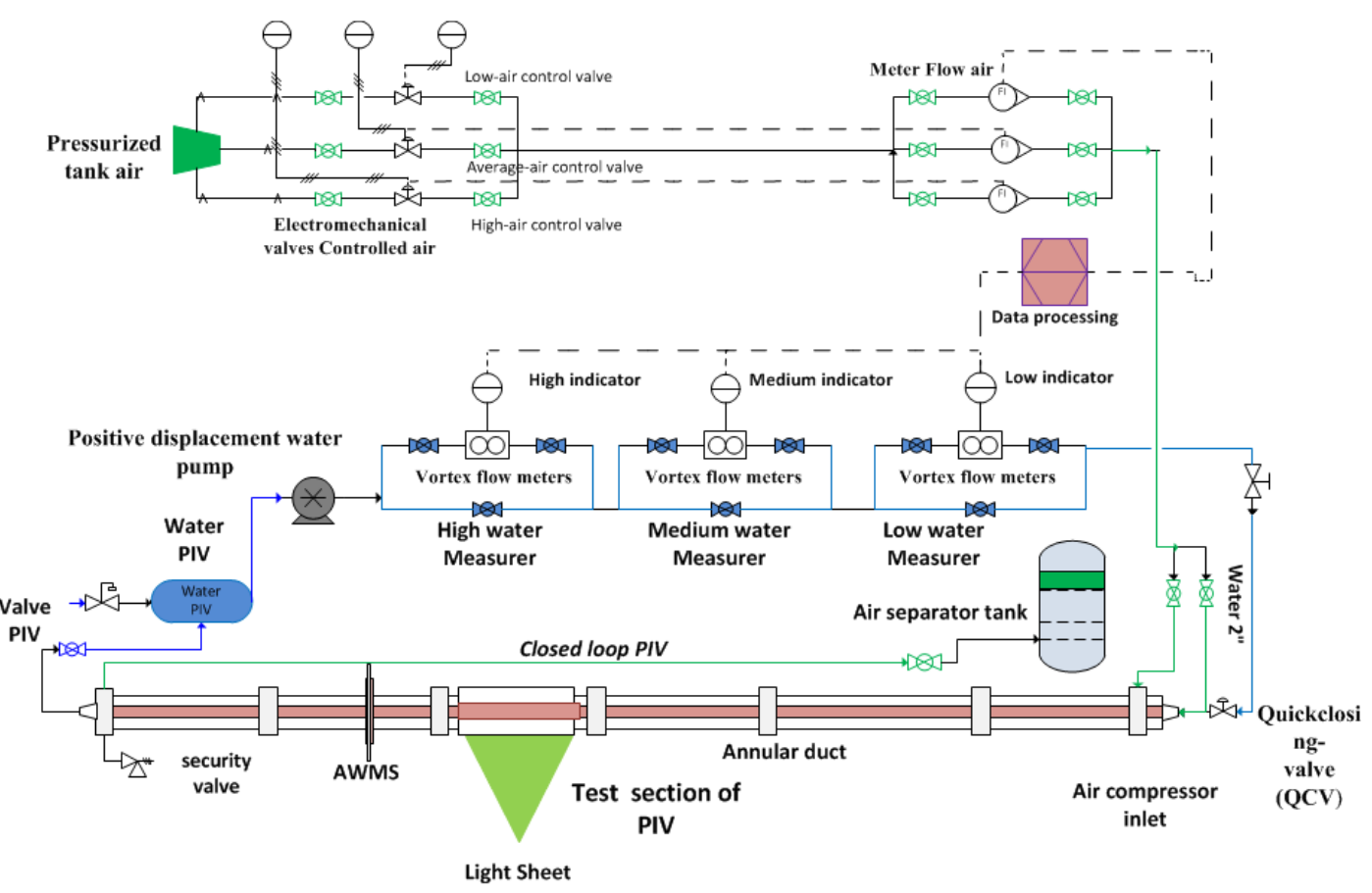




\subsection{Aparato Experimental PIV}

Uma câmera CCD (2448 pixel x 2050 pixel, 12-bit, 5 Mpx, Charge Coupled Device) foi posicionada na seção de teste captar as imagens do escoamento. $\mathrm{O}$ tamanho da partícula traçadora foi aproximadamente $10 \mu \mathrm{m}$ (esferas ocas de vidro), a iluminação era fornecida por um laser verde de duplo pulso de elevada potência $(70 \mathrm{~mJ} /$ pulso). $\mathrm{O}$ alinhamento do plano de luz é ajustado tal que o centro do duto anular seja iluminado. O laser age como um flash fotográfico para a câmera digital. No presente estudo foi realizado o PIV do tipo duplo frame, onde são captadas duas imagens com um intervalo de tempo determinado (escolhido no software) e apropriado, de acordo com a vazão do fluido, para que se possa ter um deslocamento de partícula mais adequado (em torno de 5 pixels) para a obtenção do perfil de velocidades, como em (Van Doorne e Westerweel, 2007). O processamento para a obtenção dos campos de velocidade foi feito com software DaVis 8, da LaVision. Posteriormente os dados foram exportados, para serem processados em MatLab. Nos escoamentos laminares monofásicos foram adquiridas 500 imagens, enquanto para os turbulentos 4000, os quais precisam de um número maior de imagens, para a que as estatísticas de turbulência convirjam. Para os escoamentos bifásicos, os resultados mostrados são preliminares, onde foram adquiridas 3000 imagens.

\section{RESULTADOS}

\subsection{Escoamento Monofásico Laminar}

$\mathrm{Na}$ figura 2 foi feito uma comparação entre os perfis de velocidades analítico e experimental. O escoamento foi conduzido com uma vazão de 7,5 L/min, $\mathrm{Re}=740$, para que estivesse completamente desenvolvido na seção de testes, onde o número de Reynolds deveria ser menor que 810. O perfil analítico foi obtido através das equações (1) e (2), tiradas de (B. R. Munson et al., 2004):

$$
\begin{aligned}
& u_{a}(r)=-\frac{\Delta P}{L \cdot 4 \mu}\left[r^{2}-r_{\text {max }}{ }^{2}+\frac{r^{2}-r_{\text {max }}^{2}}{\ln \frac{r_{\operatorname{mx}}}{r_{\min }}} \ln \frac{r}{r_{\max }}\right] \\
& \frac{\Delta P}{L}=\frac{8 Q \mu \ln \frac{r_{\text {max }}}{r_{\min }}}{\pi\left[\ln \frac{r_{\text {max }}}{r_{\text {min }}}\left(r_{\text {max }}^{4}-r_{\text {min }}^{4}\right)-\left(r_{\text {max }}^{2}-r_{\text {min }}^{2}\right)^{2}\right]}
\end{aligned}
$$

Onde: $\frac{\Delta P}{L}$ é a queda de pressão por unidade de comprimento, $\mathrm{Q}$ é a vazão do fluido, $\mathrm{r}_{\text {min }}$ e $r_{\max }$ são os raios do tubo interno e externo, respectivamente, $r$ é a posição radial e $\mu$, a viscosidade do fluido. Devido à baixa magnitude de $\frac{\Delta P}{L}$, ela foi calculada pela equação (2), para se obter o perfil de velocidades pela equação (1). $O$ erro do perfil experimental foi estimado através da equação (3), obtendo um valor médio de 4,4\%, máximo e mínimo de $6,1 \%$ e $1,2 \%$; respectivamente. 


$$
e(\%)=100 \frac{\left|V_{\text {pontos } P V}-V_{\text {pontosAnaticicos }}\right|}{V_{\text {pontosPIV }}}
$$

Analisando-se os perfis (Fig. 2) percebe-se que o ponto máximo de velocidades está mais próximo do tubo interno (representando pela posição adimensional ' 1 ', enquanto que o tubo externo é representado por ' 0 '), vê-se também, que o perfil experimental do PIV é um pouco deslocado em relação ao analítico, isso é devido ao fato que, um pequeno desalinhamento nos espelhos que refletem o laser para direcioná-lo a região desejada, pode causar um grande erro na medida de um perfil laminar, visto em (Van Doorne e Westerweel, 2007), (Cely et al. 2017) esse efeito é menos significativo nos turbulentos.

Figura 2 - Comparação de perfil de velocidades: Analítico e Experimental

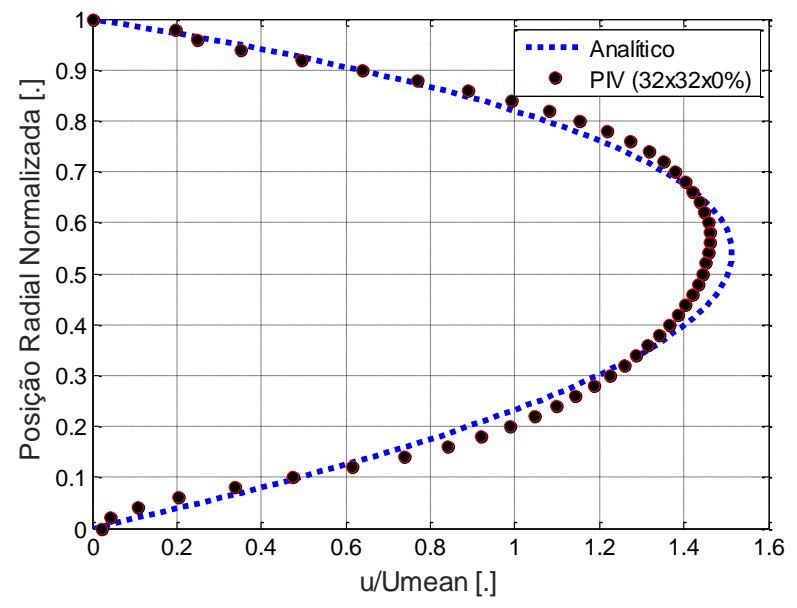

O objetivo da medição do perfil laminar era para a validação da bancada experimental, foram obtidos dados confiáveis a $0,9 \mathrm{~mm}$ da parede externa. Tal objetivo foi atingido, devido aos pequenos erros relativos encontrados.

\subsection{Escoamento Monofásico Turbulento}

Escoamentos monofásicos turbulentos foram analisados, esperava-se um perfil achatado, com ponto de máximo mais próximo do tubo interno, em analogia ao escoamento laminar. A figura 3 mostra perfis para seis escoamentos diferentes, variando-se as velocidades superficiais de $0.075 \mathrm{~m} / \mathrm{s}$ a $0.215 \mathrm{~m} / \mathrm{s}$, consequentemente o número de Reynolds de $7106 \mathrm{a}$ 20431, bem como as estatísticas de flutuações das velocidades e os cross-moments (basta multiplica-los pela densidade da água e tem-se as Tensões de Reynolds). Percebem-se semelhanças na forma dos perfis de velocidades: o perfil para $0.215 \mathrm{~m} / \mathrm{s}$ mostra velocidades de aproximadamente 2 vezes àquelas do perfil de $0.110 \mathrm{~m} / \mathrm{s}$. As Tensões de Reynolds são crescentes com o número de Reynolds, como é visto na literatura para escoamentos em tubos em (den Toonder e Nieuwstadt, 1997). Os erros nas medições são mais difíceis de serem quantificados, devido à não existência de modelo analítico para tal tipo de escoamento em duto anular, porém, nos desvios na velocidade radial $\left(\mathrm{V}_{\mathrm{rms}}\right)$, são encontrados picos muito próximos às paredes do duto (entre as posições radiais 0.95 e 0.98 , correspondendo a $1 \mathrm{~mm}$ e 2,5 mm do tubo interno) para os escoamentos de maiores números de Reynolds, algo que não 
deveria ocorrer devido à condição de não deslizamento da Mecânica dos Fluidos, (Schlichting and Gersten 1979). Esse é um tipo de erro presente na técnica PIV, devido a refrações e reflexões do laser na parede do duto, fazendo com que as medidas nessas regiões sejam mais incertas (Van Doorne e Westerweel, 2007).

Figura 3 - Perfis de Velocidade e estatísticas de turbulência
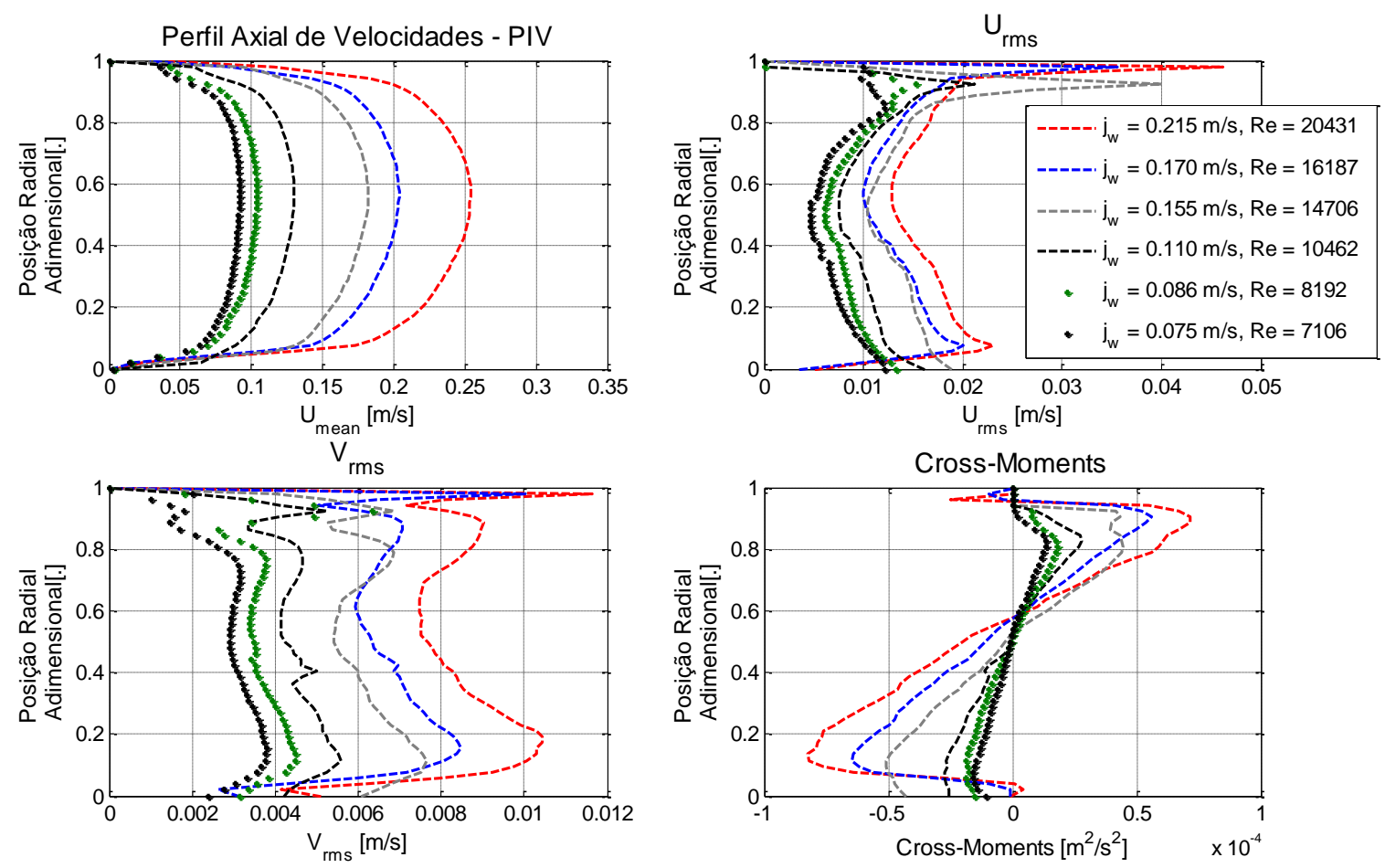

\subsection{Escoamento Bifásico}

Foi analisado um escoamento bifásico com a seguinte configuração: $\mathrm{j}_{\mathrm{w}}=0.152 \mathrm{~m} / \mathrm{s}$ e $\mathrm{j}_{\mathrm{a}}=0.039 \mathrm{~m} / \mathrm{s}$. Devido à complexidade do padrão de escoamento encontrado (pistonado), as imagens obtidas pela câmera do PIV necessitavam de uma divisão em grupos: imagens que captaram a bolha inteira e imagens que captaram somente o pistão. Dessa maneira, foram obtidos perfis médios de velocidades do pistão e em diferentes regiões da bolha: nariz, meio e cauda. Na Fig. 4, é mostrado o perfil médio e o campo instantâneo de velocidades da água, no meio da bolha. Vê-se que há velocidades negativas, ocorre 'backflow'.

Figura 4 - Perfil e Campo de Velocidades no Meio da Bolha 

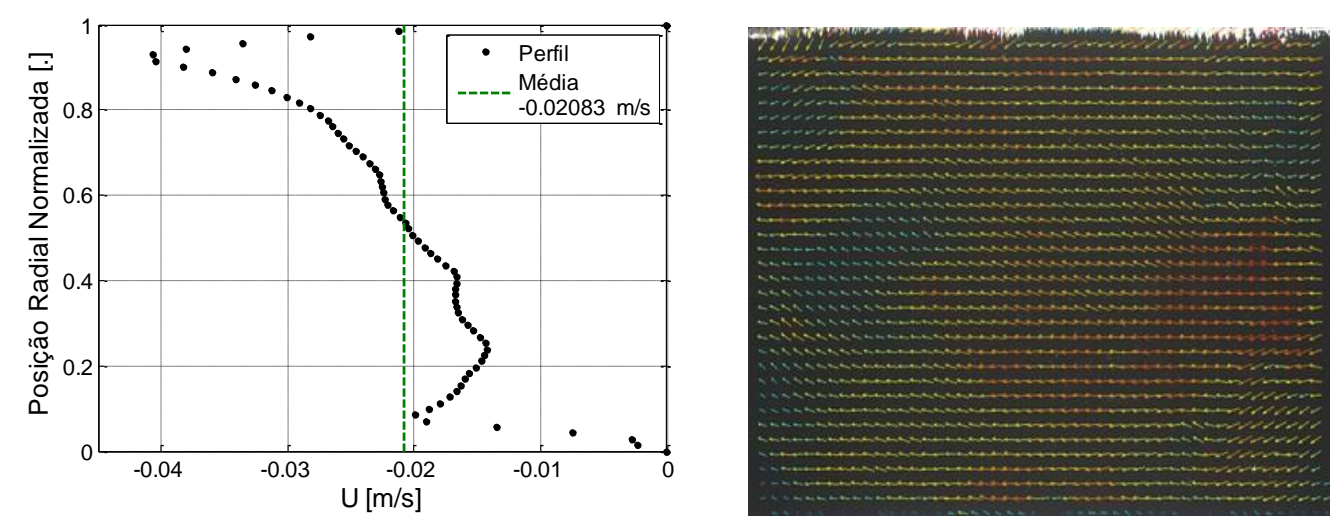

\section{CONCLUSÃO}

O trabalho aqui apresentado mostra a importância de uma boa calibração para a obtenção de um perfil de velocidades coerente nos escoamentos laminares. Quanto aos turbulentos, ficou evidente que as tensões de Reynolds têm dependência com o número de Reynolds, elas crescem conforme o número aumenta, como foi observado em tubos por (den Toonder and Nieuwstadt 1997), (Kumara et al. 2010a; Elseth 2001). O perfil de velocidades turbulento mostrou que o ponto de velocidade máxima ocorre mais próximo do tubo interno, similarmente como é visto no caso laminar. Nos escoamentos bifásicos, os resultados dão um bom entendimento do fenômeno ocorrido: com a passagem da bolha o perfil de velocidades do líquido abaixo da mesma é alterado, sofre uma desaceleração, chegando a ter valores negativos. Isso deve ter efeitos nas tensões de cisalhamento e na queda de pressão. Trabalhos similares, para efeito de comparação, em escoamentos pistonados em geometria anular, não foram encontrados na literatura.

\section{REFERÊNCIAS}

BIRVALSKI, M., M. J. TUMMERS, AND R. A W M HENKES. "Measurements of Gravity and Gravity-Capillary Waves in Horizontal Gas-Liquid Pipe Flow Using PIV in Both Phases." International Journal of Multiphase Flow 87. Elsevier Ltd: 102-13, 2016.

CELY, MARLON M. H., ANDREZA B. F. DE OLIVEIRA, VICTOR E. C. BAPTISTELLA, AND OSCAR M .H. RODRÍGUEZ. "PIV Measurements of the Instantaneous Velocities of Flow in an Annular Duct." 2017 IEEE - International Instrumentation and Measurement Technology Conference, 2017.

DOORNE, C. W H VAN, AND J. WESTERWEEL. "Measurement of Laminar, Transitional and Turbulent Pipe Flow Using Stereoscopic-PIV." Experiments in Fluids 42 (2): 25979, 2007.

E. EDGARD, D. F. YOUNG, T. H. OKUSHI. "Fundamentos Da Mecânica Dos Fluidos.", Ed. Blucher, vol. 4, 2004

ELSETH, GEIR. "An Experimental Study of Oil / Water Flow in Horizontal Pipes Department of Technology ( HiT-TF ) Telemark University College Kjølnes Ring, N3914 Porsgrunn," no. 1301: 270, 2001.

KUMARA, W. A S, G. ELSETH, B. M. HALVORSEN, AND M. C. MELAAEN. "Comparison of Particle Image Velocimetry and Laser Doppler Anemometry 
Measurement Methods Applied to the Oil-Water Flow in Horizontal Pipe." Flow Measurement and Instrumentation 21 (2). Elsevier Ltd: 105-17, 2010.

KUMARA, W. A S, B. M. HALVORSEN, AND M. C. MELAAEN. "Particle Image Velocimetry for Characterizing the Flow Structure of Oil-Water Flow in Horizontal and Slightly Inclined Pipes." Chemical Engineering Science 65 (15). Elsevier: 4332-49, 2010.

SCHLICHTING, DOCTOR HERMAN, AND K GERSTEN. "Boundary-Layer Theory." European Journal of Mechanics - B/Fluids 20: 817, 1979.

TOONDER, J M J DEN, AND F T M NIEUWSTADT. "Reynolds Number Effects in a Turbulent Pipe Flow for Low to Moderate Re." Phys. Fluids 9 (June): 3398, 1997.

WESTERWEEL, J. "Fundamentals of Digital Particle Image Velocimetry." Measurement Science and Technology 8 (12): 1379-92, 1997.

WILLERT, CHRISTIAN. "Stereoscopic Digital Particle Image Velocimetry for Application in Wind Tunnel Flows." Measurement Science and Technology 8 (12): 1465-79, 1999. 